\title{
The Value of the Cosmological Constant
}

\author{
John D. Barrow ${ }^{1}$ and Douglas J. Shaw ${ }^{2}$ \\ DAMTP, Centre for Mathematical Sciences, \\ Cambridge University, Cambridge CB3 0WA, \\ United Kingdom
}

October 22, 2018

\begin{abstract}
We make the cosmological constant, $\Lambda$, into a field and restrict the variations of the action with respect to it by causality. This creates an additional Einstein constraint equation. It restricts the solutions of the standard Einstein equations and is the requirement that the cosmological wave function possess a classical limit. When applied to the Friedmann metric it requires that the cosmological constant measured today, $t_{U}$, be $\Lambda \sim t_{U}^{-2} \sim 10^{-122}$, as observed. This is the classical value of $\Lambda$ that dominates the wave function of the universe. Our new field equation determines $\Lambda$ in terms of other astronomically measurable quantities. Specifically, it predicts that the spatial curvature parameter of the universe is $\Omega_{\mathrm{k} 0} \equiv-k / a_{0}^{2} H^{2}=-0.0055$, which will be tested by Planck Satellite data. Our theory also creates a new picture of self-consistent quantum cosmological history.
\end{abstract}

The cosmological constant, $\Lambda$, has played a stimulating role in gravitation theory ever since Einstein introduced it in 1917 to provide the gravitational repulsion needed to support a static universe. A new insight emerged in 1934 when Lemaitre [1] first showed how to reinterpret it as a Lorentz-invariant vacuum 'fluid' in Einstein's equations. More recently, this formulation has led to its interpretation as the vacuum energy density of the universe [2], $\rho_{v a c}=\Lambda / 8 \pi$, as Lemaitre suggested, [3, 4]. Before 1998, there was no direct astronomical evidence for $\Lambda$ and the observational upper bound was so strong $-\Lambda<10^{-120}$ Planck units that many particle physicists suspected that some fundamental principle must force its value to be precisely zero.

Alas, no such principle was forthcoming. Worse still, any attempt to set $\Lambda$ to zero at the start of the universe was overcome by the generation of large effective $\Lambda$ values when the universe cooled through phase transition in its early stages. The result was a $\Lambda$ value today at least $10^{56}$ times larger than observations permitted. Then, in 1998, two independent groups, led by Riess and Perlmutter [5] used Type 1a supernovae to show that the universe is accelerating. This discovery provided the first direct evidence that $\Lambda$ is non-zero, with $\Lambda \sim$ $1.7 \times 10^{-121}$ Planck units.

This remarkable discovery highlighted the question of why $\Lambda$ has this unusually small value. It is $10^{121}$ times larger than the 'natural' value for the vacuum energy of the universe. Moreover, it is very close to the largest value $\left(\sim 10^{-120}\right)$ that it could take without preventing galaxies from having formed $[6$. So far, no explanations have been offered for the proximity of the $\Lambda$ to $1 / t_{U}^{2} \sim 1.6 \times 10^{-122}$, where $t_{U} \sim 8 \times 10^{60}$ is the present expansion age of the universe in Planck time units. Attempts to explain the coincidence that $\Lambda \sim 1 / t_{U}^{2}$ have relied 
upon ensembles of possible universes, in which all possible values of $\Lambda$ are found. Anthropic selection is combined with some the prior probability distribution for $\Lambda$ over the ensemble to find the most probable value that allows galaxies to form [7. Clearly, it would be much more attractive to predict $\Lambda$ directly using a testable theory without appeal to a multiverse of possibilities. This we shall now do.

We will extend the Einstein-Hilbert variational principle for general relativity (GR) by promoting $\Lambda$ from being a parameter to a 'field' and only include causal variations which are on and inside our past light cone. In addition to the usual Einstein equations, this creates a new integral field equation which determines $\Lambda$ in terms of other properties of the observed universe. Crucially, the observed classical history always has $\Lambda \sim 1 / t_{U}^{2}$ when observed at time $t_{U}$. In GR, $\Lambda$ is a true constant and is not seen to evolve, although the constant value it takes depends on the cosmic time of observation. Hence, the resulting history is indistinguishable from GR with a constant value of $\Lambda$ put in by hand. Moreover, this theory produces a firm prediction for $\Lambda$ in terms of other measurable quantities and is testable by future observations.

Conventionally, $S_{\text {tot }}\left[g_{\mu \nu}, \Psi^{i}, \Lambda ; \mathcal{M}\right]$ is the total action of the universe defined on a spacetime $\mathcal{M}$, with boundary $\partial \mathcal{M}$. $S_{\text {tot }}$ is a functional of the metric, $g_{\mu \nu}$, and matter fields, $\Psi^{i}$, and also depends on the fixed parameters like $\Lambda$ and the fundamental constants. It is well known that the classical field equations follow from extremizing $S_{\text {tot }}$. At the quantum level, physics is determined by a partition function $Z_{\Lambda}[\mathcal{M}]$ which is a $\operatorname{sum}$ of $\exp \left(i S_{\text {tot }}\right)$ over all configurations of the fields with parameters such as $\Lambda$ fixed:

$$
Z_{\Lambda}[\mathcal{M}]=\sum_{g_{\mu \nu}, \Psi^{i}} e^{i S_{\mathrm{tot}}}
$$

The dominant configurations, or histories, are those in which the classical field equations hold.

Our new proposal for solving the $\Lambda$ problem requires only a simple modification of this standard variational principle. When we promote $\Lambda$ from a fixed parameter to a field that can take many possible values, all of which contribute to the partition function, we also demand that the action and the partition function are causal, so they only depend only field configurations in the observer's causal past. This preserves classical causality.

This promotion of $\Lambda$ from fixed parameter to one that can take many values occurs in some theories of quantum gravity. In string theory, $\Lambda$ can take many possible values over a continuous, or tightly-spaced discrete spectrum. There may be $O\left(10^{500}\right)$ different vacua and $\Lambda$ values with a spacings of $O\left(10^{-500}\right)$ in Planck units. Provided this spacing is tighter than $\Delta \Lambda=\left(\delta^{2} S_{\text {tot }} / \delta \Lambda^{2}\right)^{-1 / 2}$, we can approximate the discrete spectrum by a continuous one. In Planck units, $\Delta \Lambda \sim \Lambda H$, and so for the observed universe, where $H^{2} \sim \Lambda \sim 10^{-122}$, $\Delta \Lambda \sim 10^{-186}$.

When we $S_{\text {tot }}$ with respect to the matter and metric fields we get the usual Einstein equations, but extremizing $S_{\text {tot }}$ with respect to $\Lambda$ gives a new field equation, $\delta S_{\text {tot }} / \delta \Lambda=0$, equivalent to

$$
\frac{\mathrm{d} S_{\mathrm{cl}}(\Lambda)}{\mathrm{d} \Lambda}=0
$$

where $S_{\text {cl }}(\Lambda)$ is $S_{\text {tot }}$ evaluated with the matter and metric fields obeying their classical field equations. Eq. (11) is an additional field equation that links $\Lambda$ to the other properties of the observable universe. Eq. (11) must hold if the observable universe is to display an approximately classical evolution, but there is no guarantee that a solution of the other Einstein equations will also satisfy Eq. (1). 
Since Eq. (11) restricts the possible classical universes, it leads to testable predictions. When evaluated for our universe, we will find that the observed value of $\Lambda$ determines the spatial curvature. There is also now a simple argument for why $\Lambda \sim t_{\mathrm{U}}^{-2}$ is natural. Equation (1) is equivalent to

$$
\int_{\mathcal{M}}|g|^{\frac{1}{2}} \mathrm{~d}^{4} x=\frac{1}{2} \int_{\partial \mathcal{M}}|\gamma|^{\frac{1}{2}}\left[N^{\mu \nu} \mathcal{H}_{\mu \nu}+\Sigma_{a} \mathcal{P}^{a}\right] \mathrm{d}^{3} x
$$

The left-hand side is just the 4 -volume, $V_{\mathcal{M}}$, of our spacetime $\mathcal{M}$. The right-hand side is a 'holographic' term defined on the boundary (of area $A_{\partial \mathcal{M}}$, say). Now, $N^{\mu \nu} \mathcal{H}_{\mu \nu}=$ $N^{\mu \nu} \delta \gamma_{\mu \nu} / \delta \Lambda \sim O\left(\Lambda^{-1} \operatorname{tr} N\right)$ and $\Sigma_{a} \mathcal{P}^{a}$ is of similar order of magnitude or smaller. Cosmologically, $\operatorname{tr} N \sim O(H)$ where $H$ is the Hubble rate (with $H\left(t_{U}\right) \equiv H_{0}$ today), and so the right-hand side of Eq.(2) is $O\left(\Lambda^{-1} H_{0} A_{\partial \mathcal{M}}\right)$. So, we expect solutions of Eq.(2) to have $\Lambda \sim O\left(H_{0}\right) A_{\partial \mathcal{M}} / V_{\mathcal{M}}$. Typically, $H_{0} \sim A_{\partial \mathcal{M}} / V_{\mathcal{M}}$ and $H_{0}^{-1}$ is determined by $t_{\Lambda}=\Lambda^{-1 / 2}$ and the age of the universe $t_{\mathrm{U}}$. Eq.(2) links the values of $t_{\Lambda}$ and $t_{\mathrm{U}}$ and, in the absence of finetunings, we predict $t_{\Lambda} \sim O\left(t_{\mathrm{U}}\right)$ and hence $\Lambda \sim O(1) t_{U}^{-2} \sim 10^{-122}$ in Planck units. If Eq.(2) admits a classical solution, then the classical value of the effective $\Lambda$ will have the observed magnitude, $O\left(t_{U}^{-2}\right) \sim 10^{-122}$, without any fine-tuning. The scale $t_{U}$ appears without fine tuning because it defines our past light cone which restricts the variations in the action to be causal.

We now apply our proposal to a Friedmann cosmology with metric:

$$
\mathrm{d} s^{2}=a^{2}(\tau)\left[-\mathrm{d} \tau^{2}+\left(1+k x^{2} / 4\right)^{-2} \mathrm{~d} x^{i} \mathrm{~d} x^{i}\right],
$$

where $k$ determines the spatial curvature. The matter is a perfect fluid with pressure $P$, and density $\rho$.

The total action is

$$
S_{\mathrm{tot}}=S_{\mathrm{EH}}+S_{\Lambda}+S_{\mathrm{GHY}}^{(u)}+S_{\mathrm{m}}+\ldots,
$$

where $S_{\mathrm{EH}}$ is the Einstein-Hilbert action, $S_{\Lambda}=-\kappa^{-1} \int_{\mathcal{M}} \mathrm{d}^{4} x \sqrt{-g} \Lambda, S_{\mathrm{GHY}}^{(u)}$ is the GibbonsHawking-York boundary term on the past-light cone boundary, $\partial \mathcal{M}_{u}$, of $\mathcal{M}$, and $S_{\mathrm{m}}$ is the matter action; the dots (...) represent boundary terms on the initial hypersurface $\partial \mathcal{M}_{I}$. We assume that all data on $\partial \mathcal{M}_{I}$ is fixed with respect to $\Lambda$ and so the variation of any $\partial \mathcal{M}_{I}$ surface terms with respect to $\Lambda$ vanishes identically [8].

We evaluate $S_{\text {tot }}$ with matter and metric fields obeying their classical field equations and find $S_{\mathrm{tot}}=S_{\mathrm{cl}}$ is

$$
S_{\mathrm{cl}}=\frac{4 \pi}{3} \int_{0}^{\tau_{0}} a^{4}(\tau)\left(\tau_{0}-\tau\right)^{3}\left[\kappa^{-1} \Gamma-P_{\text {eff }}(a)\right] \mathrm{d} \tau .
$$

The observer is at $\tau=\tau_{0}$, the total pressure is $P_{\text {eff }}=P_{\mathrm{m}}-\mathcal{L}_{\mathrm{m}}$ and

$$
\Gamma \equiv\left(k / a^{2}\right)\left[2 / 3+\tau /\left(\tau_{0}-\tau\right)\right]
$$

The dominant contributions to $P_{\text {eff }}$ come from baryons, $P_{\text {eff }} \approx-\mathcal{L}_{\text {baryons }}=\zeta_{\mathrm{b}} \rho_{\text {baryons }}$, where $\zeta_{\mathrm{b}} \approx 1 / 2$ in the chiral-bag model for baryon structure [9].

$S_{\mathrm{cl}}$ has no explicit $\Lambda$ dependence: all dependence on $\Lambda$ is encoded in the scale factor $a(\tau)$. We define $\delta \ln a / \delta \lambda=\mathcal{A}(\tau)$ where $\mathcal{A}(\tau)$ is found by perturbing Einstein's equations with respect to $\Lambda$ and requiring $\delta \ln a / \delta \Lambda=0$ initially to obtain

$$
\mathcal{A}(\tau)=\frac{a(\tau) H(\tau)}{6} \int_{0}^{\tau} \frac{\mathrm{d} \tau^{*}}{H^{2}\left(\tau^{*}\right)}
$$


where $H=a_{, \tau} / a^{2}$ is the Hubble parameter. We can now calculate $\mathrm{d} S_{\mathrm{cl}} / \mathrm{d} \Lambda=0$ and find that

$$
k=\frac{\kappa \int_{0}^{\tau_{0}}\left(\tau_{0}-\tau\right)^{3} a^{4} \zeta_{\mathrm{b}} \rho_{\mathrm{b}} \mathcal{A}(\tau) \mathrm{d} \tau}{\int_{0}^{\tau_{0}} a^{2}(\tau)\left(\tau_{0}-\tau\right)^{2}\left(4\left(\tau_{0}-\tau\right)+6 \tau\right) \mathcal{A}(\tau) \mathrm{d} \tau} .
$$

The right-hand side is positive and so only universes with $k>0$ can obey Eq. (11) and possess a classical limit in a quantum cosmology.

Here, $k$ is the average spatial curvature within the past light cone, so $k>0$ applies to the observable universe - not the whole-space time - and does not require a closed global topology. For fixed $k$ and $\tau_{0}$ (and fixed initial conditions for the matter), Eq. (3) is an implicit equation for $\Lambda$ and predicts a relation between $\Lambda, k$ and $\tau_{0}$. If we measure $\Lambda$ and $\tau_{0}$ we can predict the spatial curvature $k=k(\Lambda)$. For fixed $\tau_{0}$, increasing $\Lambda$ requires smaller $k$ and for fixed $k, \Lambda$ decreases as $\tau_{0}$ increases.

Astronomers have measured our observation time, $\tau_{0}$, and the value of $\Lambda$, but only have bounds on $k$. It is usual to express $k$ by the dimensionless parameter $\Omega_{\mathrm{k} 0} \equiv-k / a_{0} H_{0}^{2}$. For our universe, taking $\Omega_{\Lambda 0}=0.73$, and a baryon density $\Omega_{\mathrm{b} 0}=0.0423$, and CMB temperature $T_{\mathrm{CMB}}=2.725 \mathrm{~K}$, with $\zeta_{\mathrm{b}}=0.5$, we predict:

$$
\Omega_{\mathrm{k} 0}=-0.0055 .
$$

This is consistent with the current $95 \%$ CI of $\Omega_{\mathrm{k} 0} \in(-0.0133,+0.0084)$ [10]. Soon, data taken by the Planck CMB satellite, together with constraints from baryon acoustic oscillations and $H_{0}$,will test this precise prediction of $\Omega_{\mathrm{k} 0}$.

There are other wider consequences of our scenario. At any given location and time, the wave function of the universe is dominated by a classical history in which $\Lambda$ takes a single constant value. Hence, no classical time-evolution of $\Lambda$ can be observed. Yet the history that dominates, and its associated $\Lambda$ value, changes at different observation times. We see a history with $\Lambda=\Lambda_{1}$, but an observer in our past would see a different history with $\Lambda=$ $\Lambda_{2}>\Lambda_{1}$. For measurements of $\Lambda_{1}$ and $\Lambda_{2}$ to be compared, information would have to be sent from one history to another. At the level of classical physics this cannot be done. Observers will see a history consistent with the constant $\Lambda$ given by Eq.(2) (11]. Crucially, this includes registering all previous measurements of $\Lambda$ as being consistent with $\Lambda=\Lambda_{1}$. Therefore, we do not see the past as an observer in the past would see it [12].

Our simple extension of Einstein's theory therefore has striking consequences: it explains the observed value of $\Lambda$, predicts the curvature parameter of the universe, and paints a new picture of quantum cosmological history.

\section{References}

[1] G. Lemaître, Proc. Nat. Acad. Sci. 20, 12 (1934).

[2] We use Planck units in which we set $G=c=\hbar=1$.All times are therefore given in units of the Planck time: $t_{p l}=\left(G \hbar / c^{5}\right)^{1 / 2}=5.4 \times 10^{-44} s$.

[3] Y.B. Zeldovich, JETP Lett. 6, 316 (1967).

[4] S. Weinberg, Rev. Mod. Phys. 61,1 (1989); R. Bousso, Gen. Rel. Gravitation 40, 607 (2008). 
[5] A. G. Riess et al. [Supernova Search Team Collaboration], Astron. J. 116, 1009 (1998); S. Perlmutter et al. [Supernova Cosmology Project Collaboration], Astrophys. J. 517, 565 (1999).

[6] J.D. Barrow and F.J. Tipler, The Anthropic Cosmological Principle, Oxford UP, Oxford (1986), chap. 6.9.

[7] S. Weinberg, Phys. Rev. Lett 59, 2607 (1987); G. Efstathiou, Mon. Not. R. astron. Soc. 274, L73 (1995). See for example, J. Garriga and A. Vilenkin, Phys. Rev. D 77, 043526, (2008), R. Bousso, B. Freivogel, S. Leichenauer, and V. Rosenhaus, Phys. Rev. D 83, 023525 (2011).

[8] This implies that the form of Eq. (1) is independent of this choice of the $\partial \mathcal{M}_{I}$ terms.

[9] D.J. Shaw and J.D. Barrow, Phys. Rev. D 83, 04351 (2010).

[10] E. Komatsu et al., arXiv:1001.4538.

[11] Classically, this movement from one history to another has no directly detectable consequences. From a quantum perspective, the wave function is dominated by a superposition of histories with a small spread in $\Lambda$ of $\Delta \Lambda=\left(\delta^{2} S_{\text {tot }} / \delta \Lambda^{2}\right)^{-1 / 2}$. This superposition could give rise to new effects if a system were sensitive to shifts of $O(\Delta \Lambda)$. However, with $\Omega_{\Lambda 0} \sim O(1), \Delta \Lambda / \Lambda \sim \Lambda^{1 / 2} \sim 10^{-60} \ll 1$ but this effect looks undetectably small.

[12] This behaviour arises as a direct consequence of having taken $\mathcal{M}$ to be the observer's causal past which, in turn, was necessary to preserve causality when $\Lambda$ was promoted from a parameter to a field. 